\title{
TH17-DRIVEN INFLAMMATION IS PRESENT IN ALL CLINICAL FORMS OF MULTIPLE SCLEROSIS; DISEASE QUIESCENCE IS ASSOCIATED WITH GATA3-EXPRESSING CELLS
}

\author{
M. SARESELLA', P. TORTORELLA', I. MARVENTANO', N. AL-DAGHRI², \\ F. PIANCONE', A. GATTI', M. GIRONI', D. CAPUTO', M. ROVARIS' \\ and M. CLERICI ${ }^{1.3}$
}

\begin{abstract}
'Don C. Gnocchi Foundation, Milan, Italy; ' College of Science, Department of Biochemistry, Biomarker Research Program, King Saud University, Riyadh, KSA; ${ }^{3}$ Department of Biomedical Sciences and Technology University of Milan, Milan, Italy
\end{abstract}

\section{Received October 14, 2012 - Accepted January 14, 2013}

Multiple Sclerosis (MS) presents in a variety of clinical forms associated with a diverse grade of neurological impairment, different prognosis and, possibly, multiple pathogenic mechanisms. Thus, whereas relapsing-remitting (RR) MS appears to be largely driven by inflammatory processes, neurodegeneration, partially independent from inflammation, drives primary progressive (PP) and secondary progressive (SP) MS. An extensive analysis of neuroinflammation in the different forms of MS was performed by evaluating immunophenotypic and functional parameters in MBP-stimulated T lymphocytes of 103 MS patients (26 benign (BE) MS, 30 RRMS, 33 SPMS and 14 PPMS) and 40 healthy controls (HC). Results showed that: i) IL-17-producing and RORC/ $\gamma$ t-expressing CD4+ T cells (TH17 lymphocytes), as well as IL-6 expressing CD14+ cell were augmented in all patients; ii) IL-22expressing cells were increased in all forms of MS with the exception of PPMS; iii) TGF- $\beta$-expressing B cells were increased only in RRMS; and iv) GATA3-, NFATc-1, IL-13-, and IL-25-expressing cells (TH2 lymphocytes) were augmented in RRMS and BEMS patients alone. Data herein indicate a pivotal pathogenic role of TH17-driven inflammation in all clinical forms of MS and suggest that control over disease (RRMS and BEMS) is associated not with lack of inflammation per se, but rather with the activation of immune-mediated anti-inflammatory mechanisms. These results could help the design of novel diagnostic and therapeutic approaches.

Multiple sclerosis (MS), a demyelinating autoimmune disease of the central nervous system, is triggered by molecular mimicry with cross-reacting and yet undefined epitopes and is associated with the activation of CD4+ TH1 and TH17 lymphocytes, $\mathrm{CD} 8+\mathrm{T}$ lymphocytes, and $\mathrm{B}$ lymphocytes, as well as myeloid dendritic cells. The activation of these cells drives the neuroinflammation that plays an important role in damaging the myelin sheath $(1,2)$.

The most common clinical phenotype of MS is the relapsing-remitting $(\mathrm{RR})$ form, which is characterized by an acute onset of symptoms and signs suggestive of neurological dysfunction, followed by complete or partial recovery. The long-term prognosis of RRMS is usually unfavorable, since patients enter the so-called secondary progressive (SP) phase of

\section{Key words: multiple sclerosis, immunology, cytokines, TH17, TH2, neuroinflammation}

Mailing address: Professor Mario Clerici M.D.,

Don C. Gnocchi Foundation ONLUS, 
the disease and accumulate irreversible neurological disability. A different pattern, primary progressive (PP) MS, is seen in patients showing a progressive course from the onset; an even rarer form of disease is benign MS (BEMS): in this case absent or minimal neurological impairment is seen years after the onset manifestation. Notably, whereas RRMS appears to be largely driven by inflammatory processes, neurodegeneration plays an important role in the chronic brain and spinal cord injury in patients with PP and SPMS.

Proinflammatory cytokines have long been suspected to play a role in the induction and pathogenesis of MS (3-5). The recent clarification of the factors involved in the differentiation pathways of antigen-stimulated CD4 $+\mathrm{T}$ cells has prompted the need to investigate such pathways in details. To summarize, firstly the TH1 lineage is promoted by IL- 12 and requires the T-bet transcription factor (TF). TH2 produce IL-25: this protein inhibits the development of TH 17 cells directly, secondarily to its boosting effect on IL-13 (6-8) -a TH2 cytokine with a suppressive ability on TH17 cells-, or via its effect on NFATc-1, thus promoting Th2 cell differentiation through GATA-3 expression in an IL-4-dependent manner (9). TH17 differentiation requires multiple cytokines including IL- 6 , TGF- $\beta$, IL-21, and IL-23, and is dependent on the RORC/ $\gamma \tau \mathrm{TF}$; in turn TH17 cells produce IL-17, IL-21 and IL-22 (10) and play an important role in inflammation and autoimmune diseases (11). Finally, TH9 lymphocytes differentiate in the presence of IL- 4 and TGF- $\beta$, and produce IL-9 $(12,13)$.

TH17 lymphocytes were shown to be involved in the pathogenesis of MS $(14,15)$. Thus, IL17 concentration is higher in MS patients with active disease in comparison to healthy subjects or individuals with quiescent disease $(16,17)$; IL-17 expression correlates with MS severity (18); high levels of IL-17 are present in both plaques and CSF of MS patients (17-19); IL-17 production correlates with the number of active plaques detected by MRI (17, 18); IL-17 generation by CNS-infiltrating T cells and glial cells is associated with phases of disease activity (17). Notably, IL-22 and IL-17, two of the cytokines produced by TH17 cells, cooperate in disrupting the blood-brain barrier (BBB) integrity by breaking tight junctions. Thus, $\mathrm{BBB}$ endothelial cells express IL-17R and IL-22R; cytokines binding to their receptors disrupt cellular gap junctions leading to an increased permeability that facilitates cell migration through the BBB (20).

The role of TH9 in this disease is less clear, even if IL-9 neutralization by monoclonal antibody delays the onset of EAE (experimental autoimmune encephalomyelitis), an animal model of MS. Moreover, the observation that IL-9R-deficient mice develop a milder form of EAE with reduced levels of TH17 cells and IL-6-producing macrophages reinforces the possible pathogenic role of IL-9 in MS (21). Finally, it was shown that IL-9 in combination with TGF- $\beta$ can promote TH17 cell differentiation, and, on the other hand, that $\mathrm{TH} 17$ cells can produce IL-9 (22).

To clarify the association of different $\mathrm{Th}$ lymphocyte subsets with the pathogenesis of MS and the different disease patterns observed in this disease, we stimulated peripheral immune cells of PP, SP, RR, and BEMS patients and healthy controls (HC) with a pool of myelin basic protein (MBP)antigenic peptides and examined cytokine and TF profiles. Results suggest that TH2 activation possibly with the contribution of TGF $\beta$-secreting B lymphocytes - draws the difference between clinical activity and lack thereof.

\section{MATERIALS AND METHODS}

\section{Patients and controls}

One-hundred-three patients with Multiple Sclerosis as diagnosed by clinical and laboratory parameters and followed by the Centro Sclerosi Multipla, Don Gnocchi Foundation in Milano, Italy were included in the study. Thirty RRMS patients (median age $=40$ years; range $=$ 20-59 years; 19 females and 11 males) were classified as being affected by stable MS (SMS). The diagnosis of SMS was confirmed by brain and spinal cord magnetic resonance imaging (MRI) with gadolinium: MRI showed no areas of enhancement at the time of enrolment. Median disease duration was 7 years (range: $1-29$ years); the median Kurtze Expanded Disability Status Scale (EDSS) score was 1,5 (range: 1-6). Fourteen patients (median age $=50$ years; range $=37-64$ years; 6 females and 8 males) has a diagnosis of PPMS as confirmed by the typical progressive clinical course and by brain and spinal cord MRI with gadolinium that showed a particular involvement of spinal cord with many lesions but no enhancement at the time of enrolment. Median disease 
duration was 12 years (range: 3-15 years); the median EDSS was 6 (range: 4-7). Thirty-tree patients (median age $=49$ years; range $=32-67$ years; 21 females and 12 males) were diagnosed as being affected by SPMS on the basis of the clinical history and of brain and spinal cord MRI with gadolinium that evidenced a stability of the lesion load at the time of enrolment. Median disease duration was 20 years (range: 6-35 years); the median EDSS was 6,5 (range: 4,5-8). Finally, 26 patients (median age $=45$ years; range $=36-61$ years; 18 females and 8 males) were affected by BEMS as confirmed by brain and spinal cord MRI with gadolinium: MRI showed stability or in many cases an improvement of the lesion load at the time of enrolment. BEMS was determined to be present based on the most widely accepted definition, i.e. an Expanded Disability Status Scale (EDSS) score of 3.0 or less (meaning that the patient is fully ambulatory, but may have moderate disability in one functional system or mild disability in up to four functional systems), after 15 years or more from the clinical onset of disease

Median disease duration was 21 years (range: 15-30 years); the median Kurtze Expanded Disability Status Scale (EDSS) score was 2 (range: 0-3). None of the patients had received immunosuppressive drugs in the year prior to the study period; all of them gave informed consent according to a protocol approved by the local ethics committee of the Don Gnocchi Foundation. Finally a group of 40 sex- and age-matched healthy controls $(\mathrm{HC})$ (median age $=48$ years; range $33-62 ; 27$ females and 13 males) were enrolled as well in the study.

\section{Blood sample collection and cell separation}

Thirty milliliters of whole blood was collected in vacutainer tubes containing ethylenediaminetetraacetic acid (EDTA) (Becton Dickinson \& Co., Rutherford, NJ, USA). PBMC were separated on lymphocyte separation medium (Organon Teknika Corp., Durham, NC, USA) and washed twice in PBS. Viable leukocytes were determined using a Scepter ${ }^{\mathrm{TM}}$ Handheld Automated Cell Counter (Millipore, MA, USA).

\section{Synthesis of the MBP peptides}

Thirty-one HLA I restricted and 7 HLA II restricted promiscuous peptides partially overlapping and spanning the whole MBP were used to evaluate MBP-specific responses; two 9 mer MBP peptides and one 15 mer MBP peptide with the lowest score were used, respectively as negative controls for HLA I and HLA II MBP restricted responses. All these peptides have been previously used to asses MBP- specific immune responses (23). To reassess the optimal doses needed to stimulate cells, preliminary experiments were performed with PBMC from 5 patients with stable disease and from 5 healthy controls. CFSE proliferation assay in the absence of stimulation or in the presence of different doses $(10,50$ and $100 \mu \mathrm{g} / \mathrm{ml})$ of either the MBP peptide pools or the peptide control pool confirmed that proliferation of CD4+ and CD8+ T cells could be detected upon stimulation with the antigenic MBP peptides alone, indicating that data herein are indeed relative to antigen-specific T-cell clones. Peptides were synthesized using Fmoc chemistry; their purity, as assayed by HPLC, was $>70 \%$ and their composition was verified by mass spectrometry. Lyophilized peptides were dissolved at $25 \mathrm{mg} / \mathrm{ml}$ in DMSO or sterile water to prepare peptide pools $(10 \mu \mathrm{g} / \mathrm{ml}$ final concentration) (23).

\section{Stimulation of PBMC for FACS analysis}

One million PBMC were stimulated with nonimmunogenic peptides or with a pool of antigenic MBP peptides (23) $(10 \mu \mathrm{g} / \mathrm{ml})+$ anti-CD28 $\mathrm{mAb}$ (clone 37407.111; R\&D Systems $)(1 \mu \mathrm{g} / \mathrm{ml})$ to facilitate costimulation, at $37^{\circ} \mathrm{C}$ in a humidified $5 \% \mathrm{CO} 2$ atmosphere for $24 \mathrm{~h}$. For cytokine analyses, $10 \mu \mathrm{g} / \mathrm{ml}$ Brefeldin A (Sigma-Aldrich, St. Louis, MO,USA) was added to the cell cultures during the last $6 \mathrm{~h}$ of stimulation to block protein secretion.

\section{Immunofluorescent staining}

PBMC were stained with anti-CD3, -CD4, -CD19 and -CD14 monoclonal-antibodies (mAbs) (Beckman Coulter, $\mathrm{Brea}, \mathrm{CA}, \mathrm{USA}$ ) for $30 \mathrm{~min}$ at $4^{\circ} \mathrm{C}$ in the dark, washed in PBS, and treated with FIX and PERM (FIX \& PERM Cell Permeabilization kits; eBioscience San Diego, CA, USA), and incubated for $30 \mathrm{~min}$ at $4^{\circ} \mathrm{C}$ in the dark with mAbs against the following proteins: RORC/ $\gamma \tau$, T-bet, GATA3, NFkB, NFATcl, IFN- $\gamma$, IL-4, IL-6, IL-9, IL-10, IL-12p35, IL-13, IL-17a, IL-21, IL-22, IL-23p40, IL25 and TGF- $\beta$; mAbs were Fluorescein Isothiocyanate (FITC) or Phycoerythrin( PE)-conjugated.

\section{Monoclonal Abs}

The following mAbs were used: PhycoerythrinCyanin-5 (PC-5) labeled anti-CD3 (clone UCHT1) (mouselgG1) and Phycoerythrin-Cyanin-7 (PC-7)labeled anti-CD4 (clone SFCI12T4D11) (mouse IgG1), or anti-CD19- PC-5 (clone J4.119) (mouse IgG1) -or antiCD14- PC-5(clone 116), (Beckman-Coulter, Fullerton, $\mathrm{CA}$, USA). The intracellular molecule detection mAbs used were: anti-human IL-10- PE (clone JES9D7; mouse IgGl isotype; Caltag Laboratories), anti-human IL-4FITC (clone MP4-25D2, rat IgG $1_{k}$ isotope, eBioscience Cornerstone Court West, San Diego, CA, USA), antihuman TGF- $\beta$ - PE (clone 9016, mouse IgG1 isotype R\&D Systems), anti-human IFN $\gamma$-PE, anti-human IL-6- FITC (clone 1936, mouse $\operatorname{IgG}_{2 \mathrm{~B}}$ isotope, R\&D Systems), antihuman IL-9- PE (clone MH9A4, mouse $\operatorname{IgG}_{2 B . k}$ isotype, 
Biolegend, San Diego, CA, USA), anti-human IL-12FITC (clone 27537, mouse IgG isotype, R\&D), antihuman IL-13- FITC (clone 32007, mouse IgGlisotype, R\&D), anti-human IL-17a-FITC (clone BL168, mouse $\lg G_{16}$ isotype, Biolegend), anti-human IL-21-PE (clone 3A3-N2, mouse $\lg \mathrm{G}_{1}$ isotype, eBioscience), anti-human IL-22-PE (clone 142928, mouse $\operatorname{lgG}_{1}$ isotype $R \& D$ ), anti-human IL-23-PE (clone C11.5, mouse $\operatorname{IgG}_{1 k}$ isotype, Biolegend), anti-human IL-25-PE (IL-17E, clone 182203, mouse IgGlisotype, R\&D), anti-mouse/human RORC/ $\gamma \tau-\mathrm{PE}$ (clone AFKJS-9, rat IgG $\mathrm{I}_{2 \mathrm{a}}$ isotype, eBioscience), anti-mouse/human T-bet- PE (clone 39D, mouse $\mathrm{IgG}_{1}$ isotype, cBioscience), anti-mouse/human GATA3-PE (clone TWAY, rat $\operatorname{lgG}_{2 B . h}$ isotype, eBioscience), antihuman NFkB-FITC (clone C-5, mouse $\operatorname{lgG}_{2 a}$ isotype. Santa Cruz Biotechnology, Santa Cruz, CA, USA), antihuman NFATcl-PE (clone H-10, mouse IgG isotype, Santa Cruz Biotechnology).

\section{Cytometric analysis}

Analyses were performed using a Beckman-Coulter Cytomics FC-500 flow cytometer equipped with a single $15 \mathrm{~mW}$ argon ion laser operating at $488 \mathrm{~nm}$ and interfaced with CXP Software 2.1. Two-hundred thousand events, $($ median $\mathrm{T}$ cells $=110.000 ;$ median $\mathrm{B}$ cells $=$ 20.000; median monocytes :70.000) were acquired and gated on Forward and Side scatter properties for lymphocyte or monocyte and on CD3 + for CD4+ T cells, considering isotype background. Green florescence from FITC (FL1) was collected through a 525-nm band-pass filter, orange-red fluorescence from PE (FL2) through a 575-nm band-pass filter, deep-red fluorescence from PC-5 (FL4) through a 670-nm band-pass filter, and blue fluorescence from PC-7 (FL5) through a 770-nm bandpass filters. Data were collected using linear amplifiers for forward and side scatter and logarithmic amplifiers for FL1, FL2, FL4 and FL5. Samples were first run using isotype control or single fluorochrome-stained preparations for color compensation. Rainbow Calibration Particles (Spherotec, Inc. Lake Forest, IL, USA) were used to standardize results.

Luminex fluorescent head human and ELISA cytokine immunoassans

Two million PBMC were incubated on 24-well plates in $1 \mathrm{ml}$ of RPMI 1640 medium (containing 10\% human serum and $2 \mathrm{mmol} / \mathrm{L}$ glutamine and penicillin/ streptomycin) in the presence of non-immunogenic peptides or of a pool of antigenic MBP peptides $(10 \mu \mathrm{g} /$ $\mathrm{ml}$ ) (Saresella et al., 2008) at $37^{\circ} \mathrm{C}$ in a humidified $5 \%$ $\mathrm{CO} 2$ atmosphere for 5 days in 24 well plates. Supernatants were collected for detection of cytokines.
Cytokine concentrations by multiplex immunoassav and ELISA immunoassay

Cytokines were determined using multiplex sandwich immunoassays with the Fluorokine Multi Analyte Profiling Kit (R\&D Systems, Minneapolis, MN, USA) according to the manufacturer's protocol; the following cytokines were included in the kit: IL-17a, IL-6, IL-10 and IFN $\gamma$. Interleukin-12p70, IL-21, IL-22, IL-23, TGF $\beta$, and IL-9 were measured by commercially available ELISA kits (Quantikine Immunoassay R\&D Systems). The concentration of cytokines was calculated using a standard curve derived from the reference cytokine concentration standards supplied by the manufacturer.

\section{Statistical analysis}

Quantitative data were not normally distributed (Shapiro-Wilk test) and are thus summarized as median and interquartile Range (IQR; 25 and 75 percentiles). Comparisons between groups were analyzed to evaluate immunological differences. Kruskal-Wallis analysis of variance was performed for each variable; Comparisons among the different groups were made using a two-tailed Mann-Whitney test performed for independent samples. Data analysis was performed using the MEDCALC statistical package (MedCalc Software bvba, Mariakerke Belgium).

\section{RESULTS}

\section{Anti-inflammatory cytokines: MBP-stimulated CD4 + T cells}

PBMC from all patients and controls were stimulated in vitro with MBP peptides or with non-antigenic peptides. Cytofluorimetric analyses were used to detect the percentage of $\mathrm{CD} 4+\mathrm{T}$ lymphocytes producing different cytokines. As expected, cytokine expression was not observed in cell cultures incubated with non-antigenic MBPpeptides. In contrast with these results, MBPstimulated IL-25- expressing CD4 + $T$ lymphocytes were augmented in RR and BEMS compared to PP, SPMS and $H C(p<0.001$ for $R R$ and $p<0.05$ for BEMS). Because IL-25 modulates IL-13 production, this cytokine was examined next. Results showed that IL-13-expressing CD4 $+\mathrm{T}$ lymphocytes were significantly increased in BEMS compared to PPMS and $\mathrm{HC}(\mathrm{p}=0.015$ in both cases) and decreased in PP compared to $S P(p=0.016)$ (Fig. 1). Finally, IL4expressing $\mathrm{CD} 4+\mathrm{T}$ cells were comparable in all groups of individuals (data not shown). 

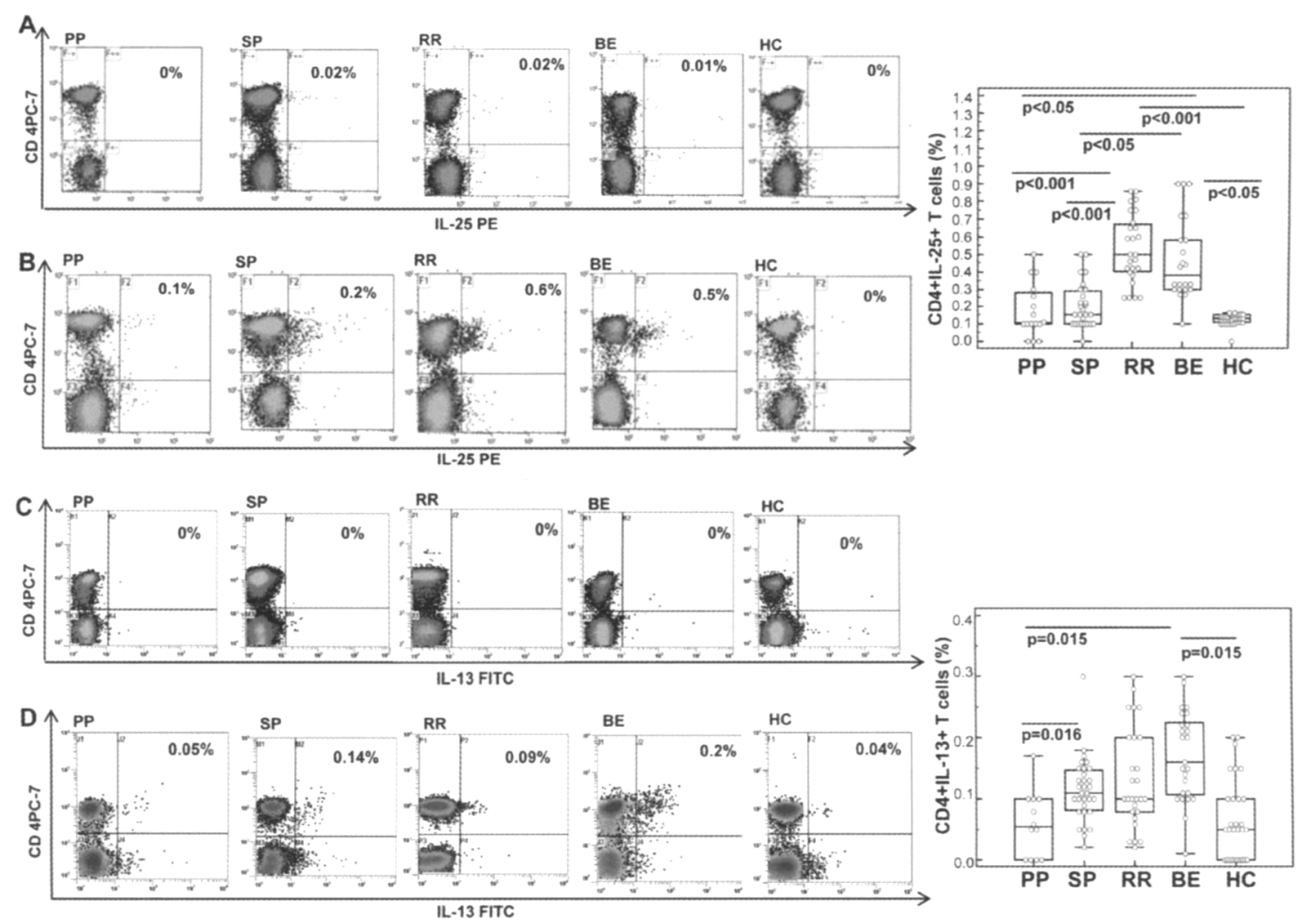

Fig. 1. Anti-inflammatory cytokines: CD4+ T lymphocytes. IL-25- and IL-13-expressing CD4+T cells in unstimulated conditions $(\boldsymbol{A}$ and $\boldsymbol{C})$ or upon stimulation with a panel of $M B P$ peptides $(\boldsymbol{B}, \boldsymbol{D})$. Representative results obtained in multiple sclerosis (MS) patients affected by primary progressive (PP), secondary progressive (SP), relapsing-remitting (RR), or benign $(B E)$ disease and in an age-and-sex-matched healthy control $(H C)$. In the upper right corner the percentage of CD4+IL13+ or CD4+IL25+ is shown. Summary results of MBP-stimulated-anti-inflammatory cytokines-producing $C D 4+T$ lymphocytes are presented in the bar graphs. The boxes stretch from the 25th to the 75 th percentile; the lines across the boxes indicate the median values; the lines stretching from the boxes indicate extreme values. Outside values are displayed as separate points. Dots represent all data. Statistical significance is shown.

$T$ lymphocytes expressing transcription factors associated with Th2 differentiation

Transcription factors (TF) involved in Th-2 differentiation were analyzed next in MBP-stimulated cells. Both NFATc-1- and GATA3- expressing CD4+ $T$ lymphocytes were augmented in BEMS compared to all other patients $(p<0.05)$ and to HC (NFATcl: $p<0.05$; GATA-3: $p<0.01$ ); GATA-3-expressing cells alone were increased in RRMS ( $p<0.05$ vs PPMS, SPMS, and $p<0.01$ vs HC) (Fig. 2). Notably, both these TF are positively regulated by IL- 25 .

$\mathrm{TH} 2$ cells play an anti-inflammatory role, these data thus suggest that in RR and BEMS a NFATc-1- and/or GATA3-driven and IL-25-mediated mechanism that hampers TH17-associated inflammation is present; this is not observed in PPMS and SPMS.

\section{Anti-inflammatory cytokines: $\quad$ MBP-stimulated CD19+ cells}

Whereas IL-10 expressing CD19+ cells were comparable in all groups of individuals, TGF $\beta$ expressing B lymphocytes were significantly increased in RRMS patients compared to SPMS $(\mathrm{p}=0.01)$ and $\mathrm{HC}(\mathrm{p}=0.001)$ (Fig. 3).

MBP-stimulated pro-inflammatory cytokines in CD4 + Tlymphocytes

MBP-stimulated IL-17-expressing CD4+ 

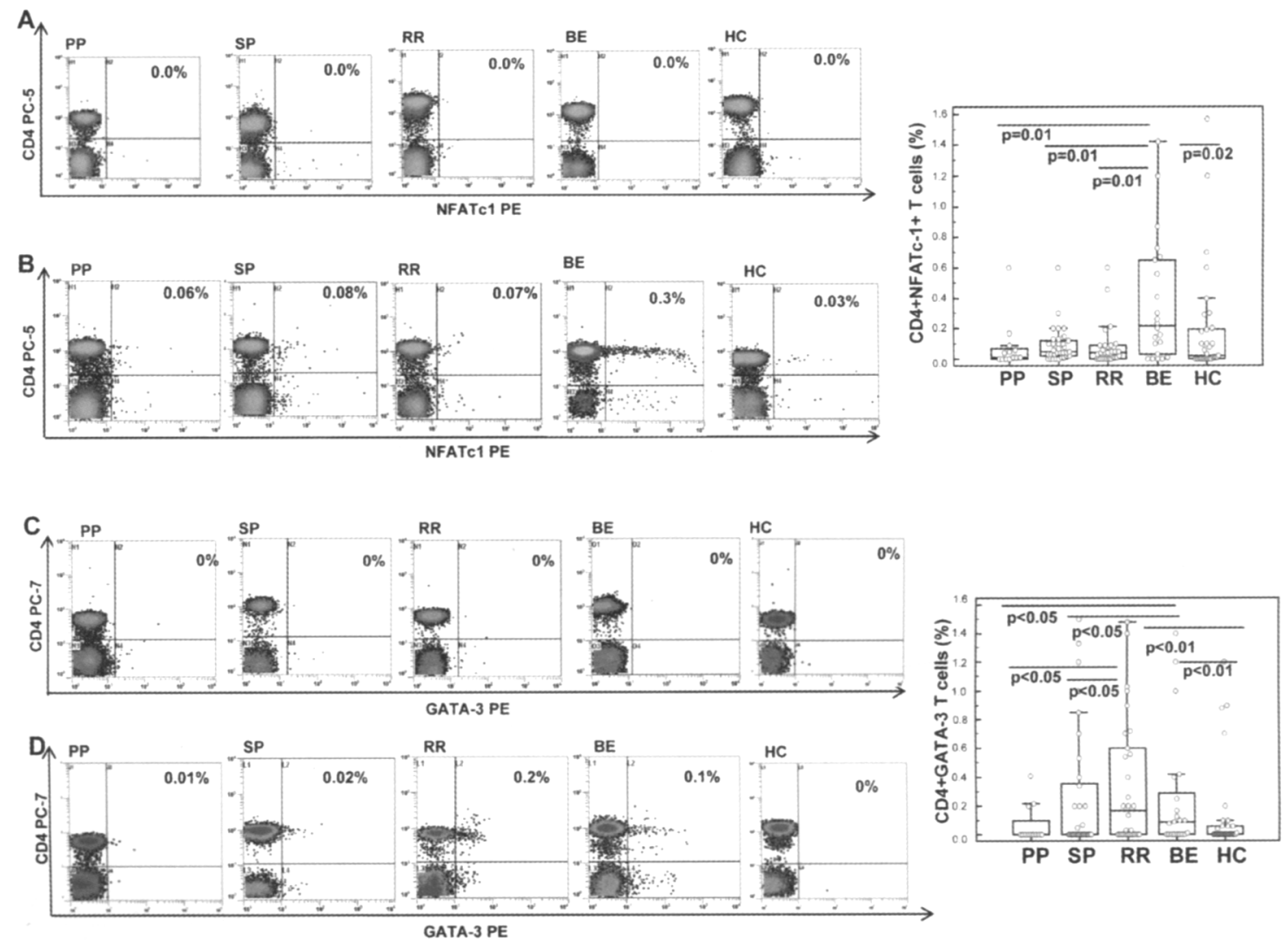

Fig. 2. Th-2 Transcription factors expressing CD4 $+T$ lymphocytes. NFATc-1 and GATA-3 expressing-CD4+ cells in unstimulated conditions $(\boldsymbol{A}, \boldsymbol{C})$ or upon stimulation with a panel of $M B P$ peptides $(\boldsymbol{B}, \boldsymbol{D})$ in $M S$ patients with different patterns of disease and healthy controls $(H C)$. In the upper right corner the percentage of CD4+NFATc- $1+$ or $C D 4+$ GATA-3 + is shown. Summary results of MBP-stimulated-TF-expressing-CD4+Tlymphocytes are presented in the bar graphs. The boxes stretch from the 25th to the 75th percentile; the lines across the boxes indicate the median values; the lines stretching from the boxes indicate extreme values. Outside values are displayed as separate points. Dots represent all data. Statistical significance is shown.

lymphocytes were significantly augmented ( $\mathrm{p}<0.001$ for all comparisons) in all groups of MS patients compared to $\mathrm{HC}$ (Fig. 4).

IL-17 is produced by TH-17 together with other cytokines including IL-21 and IL-22, these cytokines were examined next. Whereas IL-21-expressing CD4+ T lymphocytes were similar in all patients and controls, IL-22-expressing cells were significantly augmented in RRMS, SPMS, and BEMS ( $p<0.05$ for all comparison), but not in PPMS patients, compared to controls (Fig. 4). Notably, MBP-stimulated IFN $\gamma$, and IL-9- expressing CD4+ $T$ cells were similar between all groups of MS patients and when patients were compared to controls (data not shown). These results indicate that a $\mathrm{TH} 17$-driven inflammatory condition is present in all forms of MS.

$T$ lymphocytes expressing transcription factors associated with ThI and Th17 differentiation

T-bet- and RORC $/ \gamma \tau$-expressing $\mathrm{T}$ lymphocytes were analyzed next; these TF are activated during the differentiation of Th1 and Th17 cells, respectively. Results showed that $\mathrm{RORC} / \gamma \tau$, involved in the differentiation of naïve cells toward the TH17 lineage, was significantly increased in SP and RRMS compared to $\mathrm{HC}(\mathrm{p}=0.004$ and $\mathrm{p}=0.01$, respectively) (Fig. 5). CD8+ cells that expressed RORC/ $\gamma \tau$ were also augmented in all groups of MS patients compared to controls (Fig. 5, lower right corner). These cells were recently shown to produce IL-17 

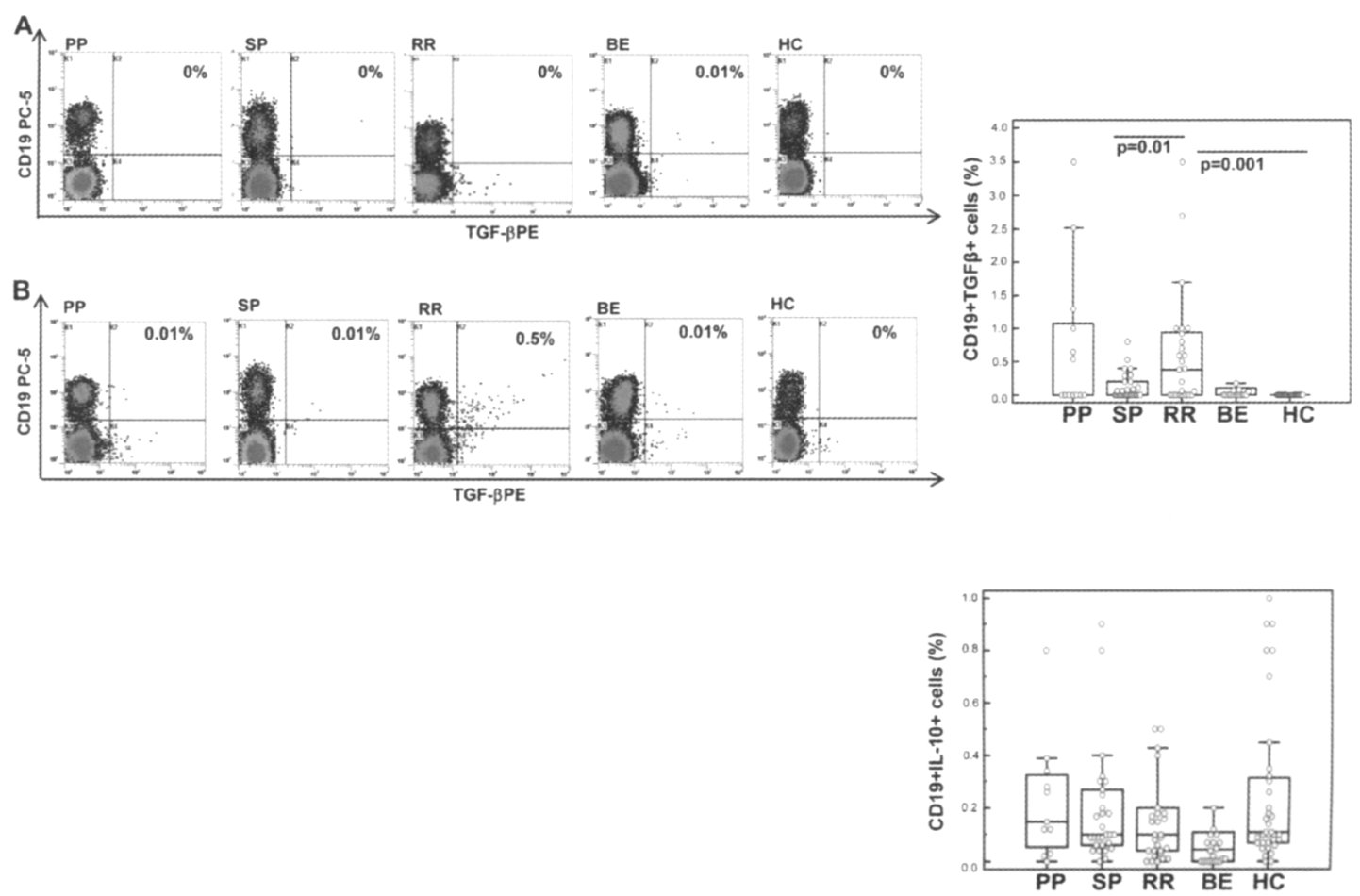

Fig. 3. Anti-inflammatory cytokines: CDI9+ cells. TGFB-expressing CD19+ cells in unstimulated conditions (A) or upon stimulation with a panel of MBP peptides $(\boldsymbol{B})$ : in MS patients with different patterns of disease and healthy controls (HC). In the upper right corner the percentage of CD19+TGF $\beta+$ cells is shown. Summary results obtained when MBPstimulated $C D 19+I L-10$-expressing cells were measured are presented in a separate panel. The boxes stretch from the 25 th to the 75th percentile; the lines across the boxes indicate the median values; the lines stretching from the boxes indicate extreme values. Outside values are displayed as separate points. Dots represent all data. Statistical significance is shown.

and were suggested to play a role in the pathogenesis of MS.

Finally, T-bet-expressing cells (T-bet is the TH1associated TF) were comparable in all the groups tested (data not shown).

Pro-inflammatory cytokines: $C D 14+$ cells in $M B P$ -stimulated PBMC

Multiple cell types mediate immune responses; to mimic as closely as possible the in vivo situation we examined cytokine-expressing CD14+ monocyte/ macrophages cells in MBP-peptides-stimulated cultures of whole peripheral blood mononuclear cells. Results indicated that IL-6- expressing CD14+ cells were significantly augmented in PP, SP, RR and BEMS patients compared to $\mathrm{HC}(\mathrm{p}=0.002, \mathrm{p}=0.002$, $p=0.04$ and $p=0.001$ respectively). Notably, IL-6expressing CD14+ cells were increased in PP and
BEMS compared to $S P$ as well $(p=0.001$ and $p=0.003$ respectively) and, finally, in BEMS compared to RRMS ( $\mathrm{p}=0.04$ ) (Fig. 6). In contrast, IL-12 and IL23-producing CD14+ cell were similar amongst all among groups analyzed (data not shown).

\section{MBP-stimulated cytokine production by PBMC}

Non-immunogenic peptides- or MBP-stimulated IL-17a, IL-6, IFN- $\gamma$, IL-10, IL-13, IL-12p70, IL-21, IL-22, TGF- $\beta$, and IL-9 production was measured next by ELISA. Results confirmed that stimulation of cells with non-immunogenic peptides does not result in the production of any cytokine. In contrast, data obtained in MBP-stimulated cell cultures showed that quantification of cytokines overall reflects the pattern seen when intracellular cytokines were measured by FACS. Therefore, IL-17, IL- 6 , and TGF- $\beta$ were increased in all forms of MS $(p<0.001$ 

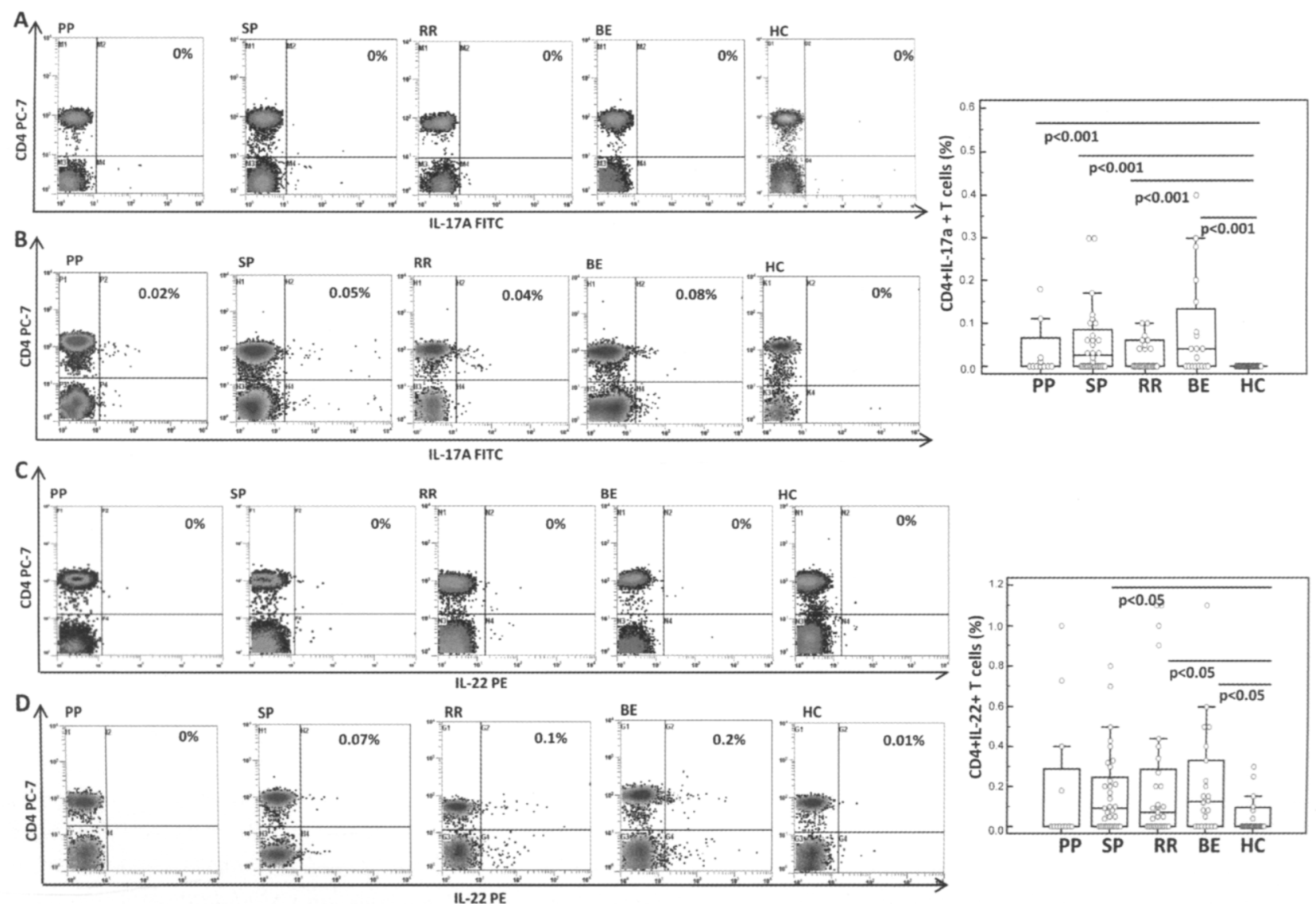

Fig. 4. Pro-inflammatory cytokines: CD4+T lymphocytes. IL-17- and IL-22-expressing CD4+ T cells in unstimulated conditions $(\boldsymbol{A}$ and $\boldsymbol{C})$ or upon stimulation with a panel of MBP peptides $(\boldsymbol{B}, \boldsymbol{D})$ in MS patients with different patterns of disease and healthy controls $(H C)$. In the upper right corner the percentage of CD4+ILI7+ or CD4+IL-22+ is shown. Summary results of MBP-stimulated-pro-inflammatory cytokines-producing CD4+Tlymphocytes are presented in the bar graphs. The boxes stretch from the 25th to the 75th percentile; the lines across the boxes indicate the median values; the lines stretching from the boxes indicate extreme values. Outside values are displayed as separate points. Dots represent all data. Statistical significance is shown.

vs HC); the highest concentration of TGF- $\beta$ was seen in RRMS; IL-22 was slightly increased in RRMS, SPMS, and BEMS; and the production of IFN- $\gamma$, IL-10 IL-12p70, IL-21, and IL-9 was similar in all analyzed groups. These results are shown in Fig. 7. Unfortunately, neither IL-13 nor IL-25 production could be measured because reagents for these cytokines are either not sensitive enough or currently not available.

\section{DISCUSSION}

To better define MBP-specific-TH cells in the diverse clinical forms of MS we analyzed such subsets in patients affected by different forms of disease. Data herein indicate that $\mathrm{TH} 17$ lymphocytes dominate MBP-specific immune response in all clinical forms of MS. TH17 cells nevertheless produce a number of cytokines, and different "functional TH17 subsets" were observed to prevail in patients affected by phenotypically-diverse forms of MS. Thus, IL17-, IL-22-, or RORC/ $\gamma \tau$ - expressing cells were increased in SP and RRMS patients, whereas cells expressing IL-17 were augmented in PPMS and in BEMS patients and IL-22 in BEMS compared to $\mathrm{HC}$.

RORC/ $\gamma \tau$ is expressed by TH lymphocytes that differentiate into $\mathrm{TH} 17$ cells. Interestingly, 

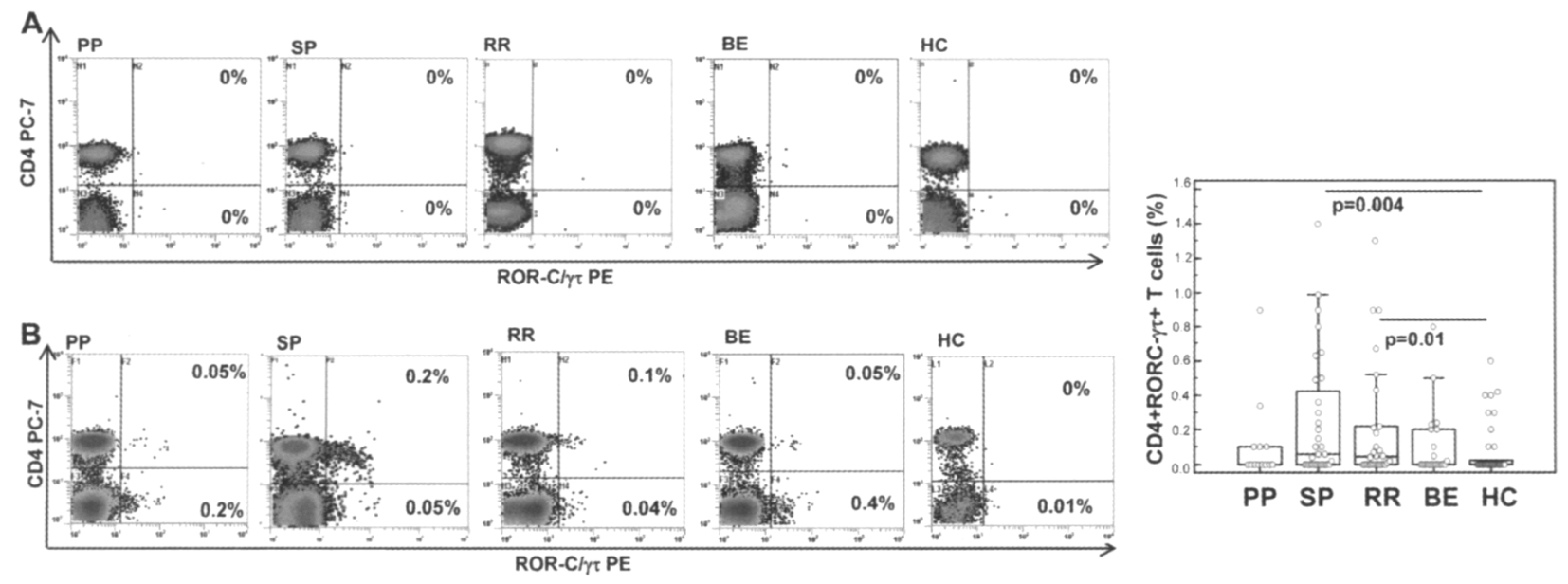

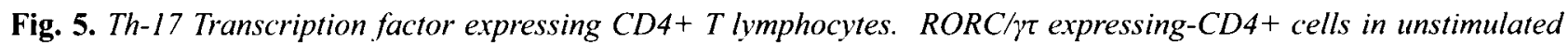
conditions $(\boldsymbol{A})$ or upon stimulation with a panel of $M B P$ peptides $(\boldsymbol{B})$ in MS patients with different patterns of disease and healthy controls $(H C)$. In the upper right corner the percentage of $C D 4+R O R-C / \gamma \tau+$ is shown. Percentages of $T C$ cells $\left(C D \dot{8}+R O R C / \gamma \tau^{+}\right)$are presented as well in the lower right corners of panels $A$ and $B$. Summary results of MBPstimulated-RORC/ $\gamma \tau+$-expressing-CD4+T lymphocytes are shown in the bar graphs. The boxes stretch from the 25 th to the 75 th percentile; the lines across the boxes indicate the median values; the lines stretching from the boxes indicate extreme values. Outside values are displayed as separate points. Dots represent all data. Statistical significance is shown.
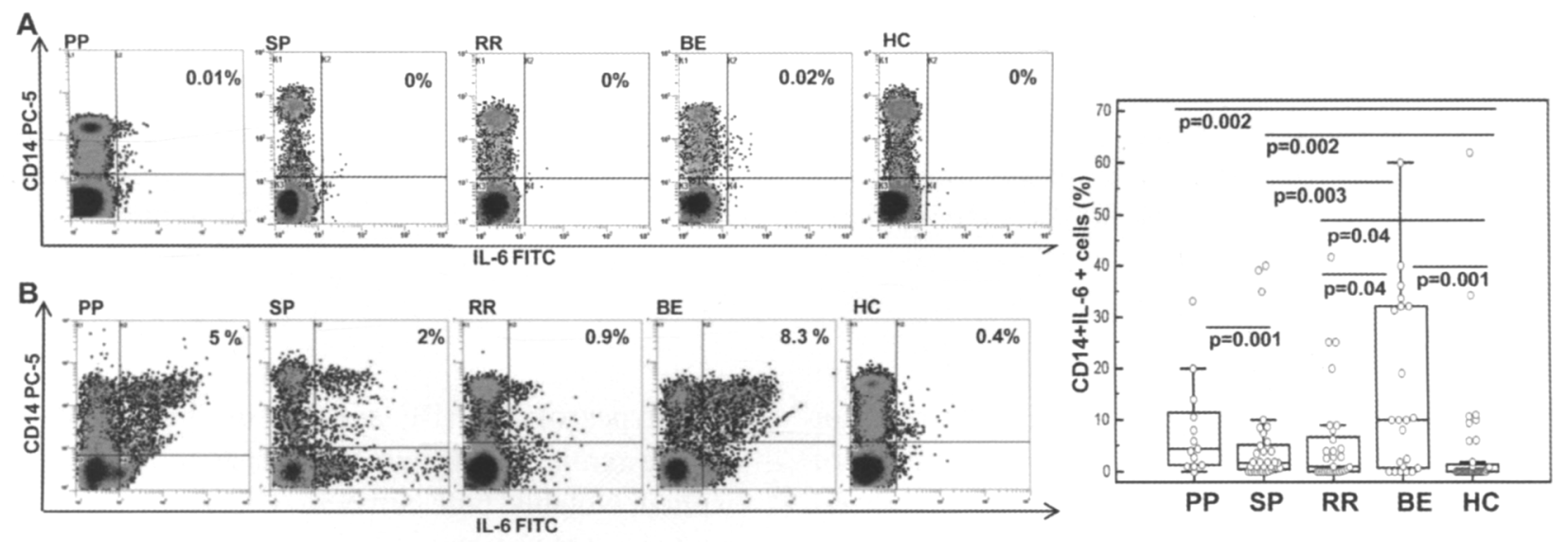

Fig. 6. Pro-inflammatory cytokines: CD14+cells. IL-6- expressing CD14+ cells in unstimulated conditions (A) or upon stimulation with a panel of MBP peptides (B) in MS patients with different patterns of disease and healthy controls $(H C)$. In the upper right corner the percentage of $C D 14+I L 6+$ is shown. The boxes stretch from the 25th to the 75th percentile; the lines across the boxes indicate the median values; the lines stretching from the boxes indicate extreme values. Outside values are displayed as separate points. Dots represent all data. Statistical significance is shown.

results showed the presence of a sizable fraction of $\mathrm{RORC} / \gamma \tau$ positive cells within the CD4-/CD8+ $\mathrm{T}$ lymphocyte population. CD8+ $\mathrm{T}$ lymphocytes include a subpopulation of cells that express $\mathrm{RORC} / \gamma \tau$ and produce IL-17. These cells, Tc17, are characterized by a reduced cytotoxic function along with low levels expression of the classical CTL markers (Tbet, Granzyme B and IFN- $\gamma$ ) (24), and are suspected to be involved in the pathogenesis of MS (18). This is based on the observations that 

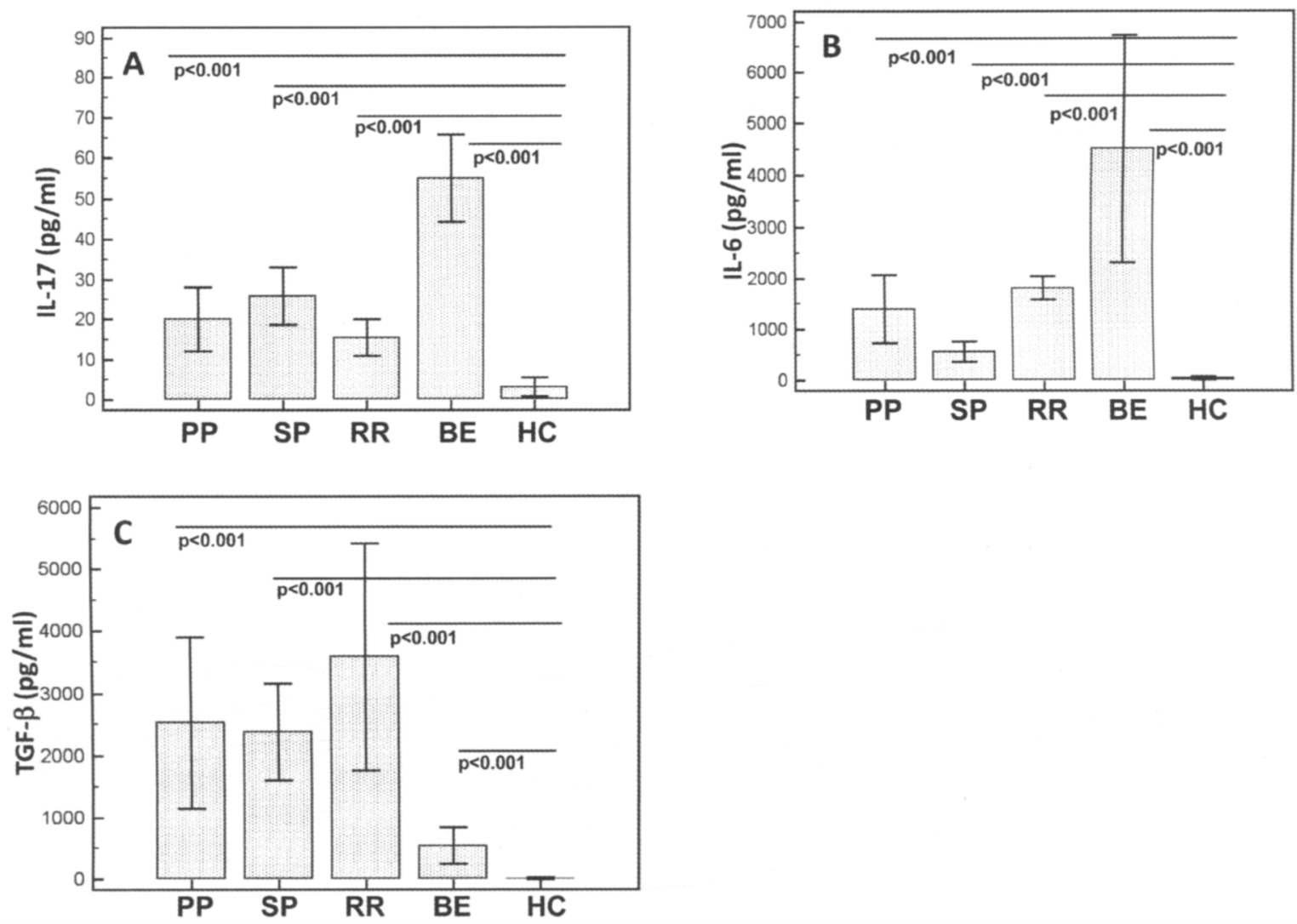

Fig. 7. MBP-stimulated cytokine production by PBMC. MBP-stimulated PBMC. IL-I7 (A),IL-6 (B), and TGF- $\beta(\boldsymbol{C})$ production $(\mathrm{pg} / \mathrm{ml})$ by cells of MS patients with different patterns of disease and healthy controls $(H C)$. Results are expressed as mean cytokine concentrations (background subtracted). Standard deviation and statistical significance are also presented.

a significant up-regulation of natural killer receptor protein $1 \mathrm{a} / \mathrm{CD} 161$ as well as increased numbers of CD161high CD8 $\mathrm{T}$ cells is seen in the peripheral blood of MS patients (25), and CD8+ T cells, and in particular CD8+ CD161+ cells can express IL22 and play an important role in the pathogenesis of autoimmunity (26). As all circulating Tc17 cells are contained within the CD161high subset, it is tempting to speculate that $\mathrm{Tc}$ do play a pathogenic role in MS (27) possibly together with other cells, including neutrophils (28).

IL-6-expressing monocyte/macrophages (MM) were significantly augmented as well in all patients. These data confirm that MBP-stimulated- IL-6 producing-CD14+ monocyte are increased in MS patients (5) and indicate that an inflammatory situation characterizes even those all clinical phenotypes of MS (BE and RR during periods of disease remission) in which disease activity is not explicit. Notably, both cell belonging to adaptive and innate immune systems over expressed inflammatory cytokines in MS, reinforcing the idea that this disease is associated with inflammation and confirming recent data indicating that MBP-specific Th-1, Th-2 and Th-17 responses correlate with disease activity (29).

Whereas TH17 cells were comparable in all clinical settings, the activation of GATA3-, NFATc-1 - IL-13- and IL-25-expressing TH2 cells was observed in RRMS and BEMS patients alone. These results suggest that control over disease is not associated with a lack of inflammation but, rather, to the triggering on anti-inflammatory, TH2-driven immune mechanisms. Such mechanisms prevail in 
RR- and BEMS patients, and are possibly responsible for the dampening of disease.

$\mathrm{TH} 2$ cells secrete a number of cytokines. Among these cytokines IL-25 and IL-13 play an important role in controlling TH17 activation: the action of IL-25 is of particular interest as this cytokine downregulates TH17 cells both directly and secondarily to its ability to increase IL-13 production. IL-25 also up-regulates NFATc-1, in turn increasing IL-4 production and enhancing GATA-3 expression and Th2 differentiation(9). Because this cytokine was augmented in RR and BEMS it is reasonable to infer that it could be responsible for disease control. Notably, NFATc-1-expressing cells were increased in BEMS alone, suggesting the presence of a stronger IL-25-mediated control mechanism on disease progression in these patients. TGF $\beta$-expressing B lymphocytes were also augmented in RRMS; these cells are suggested to functionally be Breg cells i.e. lymphocytes endowed with the ability to down-regulate immune responses (30). A number of reports dwell on the role of Treg lymphocytes in MS; to our knowledge this is the first time that Breg are suggested to also play a role in controlling disease relapses in MS patients.

Cytokines produced by MBP-stimulated PBMC were also measured; results confirmed that inflammatory cytokines belonging to both innate (IL6) and acquired (IL-17) immunity are significantly increased in all forms of MS compared to controls. Results also showed that TGF $\beta$ is overproduced in MS patients in a possible attempt to quench neuroinflammation: an attempt that is successful in RR and BEMS.

The pathogenic role played by TH17 cells in MS is likely secondary to the ability of IL-17 and IL-22 to bind specific receptors on the endothelial cells on the $\mathrm{BBB}(20,31)$; this results in the disruption of the tight junctions that characterize this barrier, allowing the penetration of monocytes and lymphocytes within the CNS. In the presence of an inflammatory milieu this facilitates neuronal death and the perpetuation of CNS inflammation through CD4+ T lymphocyte recruitment (20). The observation that IL-22 was increased in SP but not in PPMS could explain the higher-grade inflammation and active demyelination that is seen in brain plaques of SPMS (32). Notably, BBB-endothelial cells also favor the differentiation of peripheral blood-derived CD14+ monocytes into functional myeloid dendritic cells $(\mathrm{mDC})$ that can support the proliferation and expansion of $\mathrm{TH} 17$ cells (33), processes that require also the presence of IL-6. Results showing that IL-6-expressing CD14+ cells were increased in MS could explain the massive increases of TH17 lymphocytes seen in these patients.

A particular finding was that MBP-stimulated IL-6 production was greatly augmented in a subset of BEMS patients; no concurrent infections and no other inflammatory conditions were present in these individuals. One explanation stems from the suggestion that inflammatory responses might have beneficial effects in demyelinating disease. Thus, the immune process in demyelinating disease could have a beneficial permissive role in creating the conditions for remyelination and repair to take place (34).

No differences were seen in IL-9-expressing (TH9) cells, suggesting that this TH functional phenotype (13) might not play a role in the pathogenesis of MS. Nor were any differences observed either in TH1associated TF or in TH1 cytokines-secreting CD4+ or CD14 cells. These results are in contrast with previous data (35). The higher percentage of IL10-expressing compared to that of IFN $\gamma$-generating cells could justify this observation that, nevertheless, needs to be further explored.

Data herein suggest that the upregulation of $\mathrm{TH} 2$ cells seen in quiescent MS is a successful attempt by the immune response to reduce TH17 activation, its failure allowing neuroinflammation to develop. The acquisition of disease quiescence in MS might therefore not be a passive (reduction of $\mathrm{TH} 17$ cells), but rather an active (activation of $\mathrm{TH} 2$ cells) immune phenomenon. Notably, IL-25 ameliorates autoimmune diabetes (36) and exerts a protective effect in EAE (7) that is mediated by the reduction of TH 17 cells in an IL-13/IL25- and/or a NFATc-1- and GATA-3- dependent manner (6-9). These data could warrant the investigation of a possible utilization of IL-13 and IL-25 as therapeutic agents.

\section{ACKNOWLEDGEMENTS}

This study was supported by grants from "Programma Nazionale di Ricerca sull'AIDS"; 
2010-2011 Ricerca Finalizzata (Italian Ministry of Health); 2010-2011 Ricerca Corrente (Italian Ministry of Health); and 2009-2011 Fondazione Cariplo.

\section{REFERENCES}

1. Chastain EM, Miller SD. Molecular mimicry as an inducing trigger for CNS autoimmune demyelinating disease. Immunol Rev 2012; 245:227-38.

2. Nylander A, Hafler DA. Multiple sclerosis. J Clin Invest $2012 ; 122(4): 1180-8$.

3. Clerici M, Saresella M, Trabattoni D, et al. Singlecell analysis of cytokine production shows different immune profiles in multiple sclerosis patients with active or quiescent disease. J Neuroimmunol 2001; 121:88-101.

4. Ferrante P, Fusi ML, Saresella M, et al. Cytokine production and surface marker expression in acute and stable multiple sclerosis: altered IL- 12 production and augmented signaling lymphocytic activation molecule (SLAM)-expressing lymphocytes in acute multiple sclerosis. J Immunol 1998; 160:1514-21.

5. Tsunoda I, Tolley ND, Theil DJ, Whitton JL, Kobayashi H, Fujinami RS. Exacerbation of viral and autoimmune animal models for multiple sclerosis by bacterial DNA. Brain Pathol 1999; 9(3):481-93.

6. Newcomb DC, Zhou W, Moore ML, et al. A functional IL-13 receptor is expressed on polarized murine CD4+ Th17 cells and IL-13 signaling attenuates Th17 cytokine production. J Immunol 2009; 182(9):5317-21.

7. Kleinschek MA, Owyang AM, Joyce-Shaikh B, et al. IL-25 regulates Th17 function in autoimmune inflammation. J Exp Med 2007; 204:161-170.

8. Iwakura Y, Ishigame H, Saijo S, Nakae S. Functional specialization of interleukin-17 family members. Immunity 2011; 34:149-62.

9. Angkasekwinai $\mathrm{P}$, Park $\mathrm{H}$, Wang $\mathrm{YH}$, Wang $\mathrm{YH}$, Chang SH, Corry DB, Liu YJ, Zhu Z, Dong C. Interleukin 25 promotes the initiation of proallergic type 2 responses. J.Exp Med 2007; 204(7): 1509- 17.

10. Langrish $\mathrm{CL}$, Chen $\mathrm{Y}$, Blumenschein $\mathrm{WM}$, et al. IL-23 drives a pathogenic $T$ cell population that induces autoimmune inflammation. J Exp Med 2005; 201:233-40.
11. Tesmer LA, Lundy SK, Sarkar S, Fox DA. Th17 cells in human disease. Immunol Rev 2008; 223:87113.

12. Dardalhon V, Awasthi A, Kwon H, et al. IL-4 inhibits TGF-beta-induced Foxp $3+\mathrm{T}$ cells and, together with TGF-beta, generates IL-9+ IL-10+ Foxp3(-) effector T cells. Nat Immunol 2008; 9:1347-55.

13. Veldhoen M, Uyttenhove C, van Snick J, et al. Transforming growth factor-beta 'reprograms' the differentiation of $\mathrm{T}$ helper 2 cells and promotes an interleukin 9-producing subset. Nat Immunol 2008; 9:1341-46.

14. Miossec P, Korn T, Kuchroo VK. Interleukin-17 and type 17 helper T cells. N Engl J Med 2009; 361:88898.

15. Jadidi-Niaragh F, Mirshafiey A. Th17 cell, the new player of neuroinflammatory process in multiple sclerosis. Scand J Immunol 2011; 74:1-13.

16. Matusevicius D, Kivisakk P, He B, Kostulas N, Ozenci V, Fredrikson S, Link H. Interleukin-17 mRNA expression in blood and CSF mononuclear cells is augmented in multiple sclerosis. Mult Scler 1999; 5:101-104.

17. Tzartos JS, Friese MA, Craner MJ, Palace J, Newcombe J, Esiri MM, Fugger L. Interleukin-17 production in central nervous system-infiltrating $\mathrm{T}$ cells and glial cells is associated with active disease in multiple sclerosis. Am J Pathol 2008; 172:146-55.

18. Lock C, Hermans G, Pedotti R, et al. Gene-microarray analysis of multiple sclerosis lesions yields new targets validated in autoimmune encephalomyelitis. Nat Med 2002; 8:500-508.

19. Brucklacher-Waldert V, Stuerner K, Kolster M, Wolthausen J, Tolosa E. Phenotypical and functional characterization of $\mathrm{T}$ helper 17 cells in multiple sclerosis. Brain 2009; 132:3329-41.

20. Kebir H, Kreymborg K, Ifergan I, et al. Human TH17 lymphocytes promote blood-brain barrier disruption and central nervous system inflammation. Nat Med 2007; 13:1173-75.

21. Nowak EC, Weaver CT, Turner H, et al. IL-9 as a mediator of Th17-driven inflammatory disease. J Exp Med 2009; 206:1653-60.

22. Elyaman W, Bradshaw EM, Uyttenhove C, et al. IL-9 induces differentiation of $\mathrm{TH} 17$ cells and enhances function of FoxP3+ natural regulatory T cells. Proc 
Natl Acad Sci USA 2009; 106:12885-90.

23. Saresella M, Marventano I, Guerini FR, et al. Myelin basic protein-specific $\mathrm{T}$ lymphocytes proliferation and programmed cell death in demyelinating diseases. Clin Immunol 2008; 129:509-17.

24. Huber M, Heink S, Grothe H, et al. A Th17-like developmental process leads to $\mathrm{CD} 8(+) \mathrm{Tc} 17$ cells with reduced cytotoxic activity. Eur J Immunol 2009; 39:1716-25.

25. Annibali V, Ristori G, Angelini DF, et al. CD161 (high) $\mathrm{CD} 8+\mathrm{T}$ cells bear pathogenetic potential in multiple sclerosis. Brain 2011; 134:542-54.

26. Billerbeck E, Kang YH, Walker L, et al. Analysis of CD161 expression on human CD8 + T cells defines a distinct functional subset with tissue-homing properties. Proc Natl Acad Sci USA 2010; 107:3006-11.

27. Saxena A, Martin-Blondel G, Mars LT, Liblau RS. Role of CD8 $\mathrm{T}$ cell subsets in the pathogenesis of multiple sclerosis. FEBS Lett 2011; 23:3758-63.

28. Naegele M, Tillack K, Reinhardt S, Schippling S, Martin R, Sospedra M. Neutrophils in multiple sclerosis are characterized by a primed phenotype. $J$ Neuroimmunol 2012; 242(1-2):60-71.

29. Hedegaard CJ, Krakauer M, Bendtzen K, Lund H, Sellebjerg F, Nielsen CH. T helper cell type I (Thl), Th2 and Th 17 responses to myelin basic protein and disease activity in multiple sclerosis. Immunology 2008; 125:161-69.
30. Vitale G, Mion F, Pucillo C. Regulatory B cells: evidence, developmental origin and population diversity. Mol Immunol 2010; 48:1-8.

31. Beyeen AD, Adzemovic MZ, Ockinger J, et al. IL-22RA2 associates with multiple sclerosis and macrophage effector mechanisms in experimental neuroinflammation. J Immunol 2010; 185:6883-90.

32. Bramow S, Frischer JM, Lassmann H, KochHenriksen N, Lucchinetti CF, Sorensen PS, Laursen $\mathrm{H}$. Demyelination versus remyelination in progressive multiple sclerosis. Brain 2010; 133:2983-98.

33. Ifergan I, Kebir $\mathrm{H}$, Bernard $\mathrm{M}$, et al. The bloodbrain barrier induces differentiation of migrating monocytes into Th17-polarizing dendritic cells. Brain 2008; 131:785-99.

34. Hohlfeld R. Neurotrophic cross-talk between the nervous and immune systems: relevance for repair strategies in multiple sclerosis? J Neurol Sci 2008; 265:93-6.

35. Jager A, Dardalhon V, Sobel RA, Bettelli E, Kuchroo VK. Th1, Th17, and Th9 effector cells induce experimental autoimmune encephalomyelitis with different pathological phenotypes. J Immunol 2009; 183:7169-77.

36. Emamaullee JA, Davis J, Merani S, Toso C, Elliott JF, Thiesen A, Shapiro AM. Inhibition of Th17 cells regulates autoimmune diabetes in NOD mice. Diabetes 2009; 58:1302-11. 